\title{
ESTUDOS INICIAIS DE UMA ESCALA DE AVALIAÇÃO DA ANSIEDADE PARA ESTUDANTES UNIVERSITÁRIOS
}

\author{
Juliana de Oliveira Barros Amancio Mattos ${ }^{1}$ \\ Kalena Ellen dos Santos Ferraz ${ }^{2}$ \\ Sheine Suane Costa de Matos Sousa ${ }^{3}$ \\ Yasmin Carolina Lourenço de Sousa Guimarães ${ }^{4}$ \\ Thatiana Helena de Lima ${ }^{5}$
}

\begin{abstract}
RESUMO: O objetivo deste trabalho foi construir e buscar evidências de validade de estrutura interna para uma escala de ansiedade e verificar possíveis diferenças nos níveis de ansiedade para as seguintes variáveis: gênero, tipo de instituição, semestre e região de moradia. A priori, foi realizada uma busca das teorias sobre ansiedade e estabelecida uma diferenciação entre dois tipos - estado e traço - a fim de delimitar melhor o campo de análise. Participaram 214 estudantes do curso de psicologia de instituições e estados diferentes, utilizando uma ferramenta online para aplicação de uma escala de concordância de cinco pontos contendo 44 itens a serem respondidos. Nas análises pôde-se perceber uma estrutura fatorial que dividiu a escala em dois fatores, a saber, ansiedade-traço e ansiedade-estado. Para a ansiedade-estado, também houve uma subdivisão em dois fatores, sendo um relacionado às questões do contexto universitário e outro relacionado ao contexto do mercado de trabalho. Os dados foram discutidos à luz da teoria proposta para a área, bem como as limitações e sugestões foram apresentadas no texto.
\end{abstract}

Palavras-chave: Ansiedade-traço. Ansiedade-estado. Estudantes de Psicologia.

\footnotetext{
${ }^{1}$ Psicóloga, Pós- graduanda em Fundamentos da Psicanálise: Teoria e Clínica pelo Instituto ESPE. Email: julianaobamattos@gmail.com

2 Psicóloga, Especialista em Saúde da Mulher pela Universidade Federal de Pernambuco. E-mail: kalenaferraz18@gmail.com

${ }^{3}$ Psicóloga, Pós- graduanda em Psicologia Perinatal e da Parentalidade pelo Instituto Mater Online. Psicóloga em um Hospital e uma Maternidade da rede pública do Estado da Bahia. E-mail: sheinesuanes@gmail.com

4 Psicóloga, Pós-graduanda em Tradução de Inglês pela Universidade Estácio de Sá. E-mail: carolsousaguimaraes@gmail.com

${ }^{5}$ Psicóloga, mestre e doutora em Psicologia. Docente do Instituto de Psicologia da Universidade Federal da Bahia, graduação e pós-graduação. E-mail: thatianahlima@gmail.com
} 


\title{
INITIAL STUDIES OF AN EVALUATION SCALE OF ANXIETY FOR UNIVERSITY STUDENTS
}

\begin{abstract}
This work's intention was to build and search for internal structure validity evidence for an anxiety scale and to verify any possible differences on anxiety levels to the following variables: gender, institution type, school semester and dwelling region. First, a research of theories about anxiety and a differentiation between two types - state and trait - was made to better delimitate the analysis' field. 214 psychology students from different institutions and states participated, using an online tool for the application of an agreedisagree 5 -point rating scale containing 44 items to be answered. On the analysis a factorial structure which divided the scale in two factors - state and trait anxiety - could be perceived. A subdivision with two factors happened for state-anxiety, in which one is related to the university context questions and the other is related to the job market context. The data was discussed into the light of the proposed theory for the studies, as well as limitations and suggestions were presented in the text.
\end{abstract}

Keywords: Trait anxiety. State anxiety. Psychology students.

\section{ESTUDIOS INICIALES DE UNA ESCALA DE EVALUACIÓN DE ANSIEDAD PARA ESTUDIANTES UNIVERSITARIOS}

RESUMEN: El objetivo de este trabajo fue construir y buscar evidencias de validez de estructura interna para una escala de ansiedad y verificar posibles diferencias en los niveles de ansiedad para las siguientes variables: género, tipo de institución, semestre y región de residencia. Inicialmente se realizó una búsqueda de teorías de ansiedad y se estableció una diferenciación entre dos tipos, estado y rasgo, para delimitar mejor el campo de análisis. Participaron 214 estudiantes del curso de psicología de diferentes instituciones y estados, utilizando una herramienta en línea para aplicar una escala de acuerdo de cinco puntos que contiene 44 ítems para ser respondidos. En los análisis podemos ver una estructura factorial que divide la escala en dos factores, a saber, la ansiedad por rasgos y la ansiedad por estado. Para el estado de ansiedad, también hubo una subdivisión en dos factores, uno relacionado con el contexto universitario y el otro con el contexto del mercado laboral. Los datos se discutieron a la luz de la teoría propuesta para el área, así como también se presentaron limitaciones y sugerencias en el texto.

Palabras clave: Ansiedad-rasgo. Ansiedad-estad. Estudiantes de psicología. 


\section{Introdução}

A vida de universitários implica diversos fatores ansiogênicos. Ao ingressarem na universidade, os estudantes são submetidos à apreensão em relação ao futuro, cobranças pessoais, expectativas familiares, encargos financeiros, atividades domésticas, horários desordenados de sono e longas horas de estudo. Além disso, ocorrem transformações fisiológicas, neurológicas e psicológicas que decorrem da transição entre a fase de adolescência e de adulto, exigindo dos estudantes adaptação a um novo papel social (MARCHI et al., 2013).

Além de lidar com os desafios supracitados, outros aspectos característicos da rotina universitária também podem ser percebidos como estressores, quando exigirem do estudante repertório para lidar com algumas exigências, como: excesso de carga de horário de estudo, exigências no processo de formação, adaptar-se a um novo contexto, a uma nova rotina de sono e a novas demandas que exigirão estratégias de estudo e organização do seu tempo (ARINO; BARDAGI, 2018), aspectos que influenciam diretamente a rotina universitária, independentemente de o aluno estar no início, meio ou final de curso (BRANDTNER; BARDAGI, 2009).

Os termos ansiedade e estresse, de forma equivocada, muitas vezes são utilizados como sinônimos. Assim, vale apresentar uma breve definição dos conceitos aqui, deixando claro que o termo a ser utilizado neste trabalho é ansiedade e que será mais explorado adiante. A breve revisão de literatura realizada por Margis et al. (2003) aponta uma relação entre eventos estressores, estresse e ansiedade. Os autores definem o termo estressor como o evento ou estímulo que provoca ou conduz o estresse; estresse como o estado gerado pela percepção de estímulos que provocam excitação emocional, indicando uma relação etiológica entre a exposição a eventos estressores e o surgimento de sintomas e transtornos de ansiedade em geral.

Ansiedade, para Skinner (1989), é uma condição emocional complexa e aversiva que é condicionada como resultado de um emparelhamento de estímulos. As síndromes ansiosas são ordenadas em quadros em que a ansiedade é constante e permanente - ansiedade generalizada e quadros em que há crises de ansiedades abruptas, podendo ser mais ou menos intensas - crises de pânico e, de modo repetitivo, transtorno de pânico (HOLLANDER; SIMEON, 2004). De acordo com o Manual Diagnóstico e Estatístico de Transtornos Mentais DSM V (APA, 2014, p. 189), "ansiedade é a antecipação de ameaça futura", assim sendo, pode se desenvolver de tal forma, que o objeto ou situação que a desencadeia pode não ser identificado. Para 
Sadock, Sadock e Ruiz (2017), a ansiedade - que é uma resposta normal e adaptativa do organismo - passa a ser patológica quando se torna uma preocupação excessiva com vários eventos ou atividades durante um período determinado.

O Transtorno de Ansiedade Generalizada (TAG), patologia que tem afetado pessoas de todas as idades, difere do simples sintoma de ansiedade por sua intensidade e prejuízo social. Nesse caso, a ansiedade é persistente e não se restringe a um único objeto. Para diagnóstico do transtorno é necessário que os sintomas estejam presentes por mais de seis meses e tragam prejuízos sociais significativos ao sujeito. Os sintomas dominantes podem variar, por exemplo, tremores, tensão muscular, úlceras pépticas, sudorese, tontura, palpitações, cefaleia incluindo inquietação ou sensação de "nervos à flor da pele", fatigabilidade, dificuldade de concentração ou sensações de "branco" na mente, irritabilidade, tensão muscular e perturbações do sono (APA, 2014). Os indivíduos que possuem alto grau de ansiedade tendem a considerar um maior número de situações como perigosas e, consequentemente, apresentar um aumento do estado de ansiedade (CHAVES et al., 2015).

Dentre as teorias que se objetivam a explicar a ansiedade, GAMA et al. (2008) se referem à ansiedade-estado ( $\mathrm{AE}$ ) e à ansiedade-traço (AT). A primeira é entendida como um momento transitório, no qual o sujeito experimenta bastante tensão, nervosismo e apreensão por perceber certas circunstâncias como ameaçadoras. Enquanto a segunda, diferentemente, se refere a uma disposição pessoal, relativamente estável, a responder com ansiedade a situações estressantes e uma tendência a perceber um maior número de situações como perigosas.

A fim de demonstrar a importância do tema ansiedade para o contexto universitário, apresentaremos a seguir um recorte realizado dentro da literatura nacional com pesquisas relevantes publicadas nos últimos anos. Esses estudos serviram de embasamento para justificar os caminhos que levaram à nossa investigação, a saber: as variáveis coletadas inicialmente de cada participante gênero; tipo de instituição; semestre e região de moradia, e os itens construídos para que fossem respondidos pelos estudantes no que tange - a dinâmica da vida acadêmica; a formação e atuação profissional; a expectativas social e profissional relacionados à formação acadêmica.

Pela ordem cronológica, iniciaremos com o estudo de Chaves et al. (2015), que teve como objetivo investigar a relação entre a ansiedade e a espiritualidade de 
estudantes universitários da área de saúde. O estudo utilizou o Inventário de Ansiedade traço-estado (IDATE) e a Escala de Espiritualidade de Pinto e Pais-Ribeiro (2007) para coleta de dados de 609 alunos. Concluindo o seu estudo apontando a importância do desenvolvimento de estratégias de enfrentamento da ansiedade, a pesquisa aponta que os maiores níveis de ansiedade estiveram associados ao sexo feminino. Nesse sentido, considerar e estabelecer a caracterização dos participantes - sexo/gênero como variável - aparece na nossa investigação para a verificação de suas possíveis diferenças.

Cardozo et al. (2016), com o objetivo de identificar fatores associados à ocorrência de ansiedade nos acadêmicos de Biomedicina, realizaram uma pesquisa quali-quantitativa, descritiva e exploratória. $O$ estudo foi realizado com 20 acadêmicos do $7^{\circ}$ período, o que nos deu uma direção para investigarmos a variável "semestre" e as suas considerações. Sugerindo o aprofundamento da pesquisa, os autores apontam a possibilidade de ampliação dos estudos nesse contexto com: maior número de estudantes; abrangência à área de saúde e a contemplação dos diversos períodos do curso. A partir de alguns dados apresentados na pesquisa em suas tabelas: "Fatores relacionados à ocorrência de ansiedade em relação à Comunicação Profissional e Relacionamento Interpessoal" e "Fatores relacionados à ocorrência de ansiedade em relação à Formação Profissional", indícios apareceram para a construção dos itens expectativas social e profissional e formação e atuação profissional - para a nossa pesquisa, para que pudéssemos verificar suas possíveis diferenças.

Costa et al. (2017), em sua revisão de literatura, buscaram artigos a partir dos descritores "ansiedade" e "universitários" que abordassem o transtorno de ansiedade em estudantes universitários da área de saúde. Grande parte dos trabalhos encontrados utilizaram como instrumento o Inventário de Ansiedade de Beck (BAI). De acordo com os autores, a transição da adolescência para a vida adulta é uma fase marcada por mudanças biopsicossociais que levam os jovens a se defrontar com novos desafios, aliados à rotina acadêmica intensa que tornam sua saúde mental vulnerável, justificando a presente investigação e o seu arcabouço a partir do contexto dos desafios enfrentados pelos alunos em sua dinâmica acadêmica.

Medeiros e Bittencourt (2017) traçaram como objetivo analisar os motivos causadores da ansiedade em alunos que ingressaram no ensino superior. $O$ estudo foi realizado com 110 estudantes universitários da área de saúde de uma região do estado da Bahia. Desenvolvida com alunos de uma faculdade particular, a pesquisa nos 
apontou a variável "instituição" para a investigação e as suas possíveis diferenças. Ainda nesse estudo, os resultados obtidos - o grupo das mulheres que mais apresentaram dados de ansiedade em todos os graus - indicam a necessidade de se repensar o ambiente universitário, o que nos levou a construir itens no que tange ao contexto universitário.

Já Fernandes et al. (2018) buscaram evidenciar os sintomas depressivos e ansiosos em 205 alunos de um curso de enfermagem para estabelecer uma correlação com condições sociodemográficas e ocupacionais em universitários. Para avaliar os níveis de ansiedade foi utilizado o Inventário de Ansiedade de Beck (BAI). Nos resultados, foi possível observar que 62,9\% dos estudantes de enfermagem têm sintomas de ansiedade, um resultado similar ao de um estudo realizado anteriormente. Não foram encontradas associações significativas entre os níveis de ansiedade e as características sociodemográficas e ocupacionais dos entrevistados da amostra, o que nos fez pensar em pesquisar variáveis como "região de moradia", "tipo de instituição" e "gênero".

Por fim, o estudo de Bezerra et al. (2018), realizado com 166 estudantes de Psicologia de três instituições de ensino superior de Salvador, traz em seus resultados uma grande porcentagem dos participantes com níveis médios de ansiedade Traço e Estado (49\% e 54\%, respectivamente) e com níveis altos de ansiedade Traço (42\%). Para avaliar os aspectos da ansiedade dos estudantes foi utilizado o Inventário de Ansiedade Traço-Estado (IDATE). Observando esses altos índices de nível médio de ansiedade Traço e Estado em estudantes de psicologia nos questionamos sobre a importância de estudos que tragam mais dados assim sobre essa população.

Por fim, percebe-se nos estudos descritos, uma carência de instrumentos de medida que avaliem a ansiedade para esse contexto específico, sendo utilizado na maioria das vezes o BAl e o IDATE que, atualmente, estão considerados desfavoráveis pelo Sistema de Avaliação de Testes Psicológicos (SATEPSI). Dessa forma, este trabalho tem como objetivo construir uma escala de avaliação da ansiedade para universitários e buscar evidências de validade para a mesma, assim como verificar possíveis diferenças nos níveis de ansiedade para as variáveis gênero, tipo de instituição, semestre e região de moradia.

\section{Método}

\section{Participantes}


Participaram desta pesquisa 214 estudantes universitários do curso de Psicologia dos setores público e privado de seis estados do Brasil, sendo autodeclarados 168 $(78,5 \%)$ do gênero feminino e $46(21,5 \%)$ do masculino. A idade dos respondentes variou de 18 a 60 anos, com média de 25 e desvio padrão de 7,66. Com relação à instituição de ensino, 124 deles (57,9\%) estavam matriculados em instituições particulares de ensino superior e 90 deles $(42,1 \%)$ em instituições públicas, sendo 3 $(1,4 \%)$ cursando o $1^{\circ}$ semestre, $13(6,1 \%)$ o $2^{\circ}$ semestre, o $3^{\circ}$ semestre $19(8,9 \%)$, o $4^{\circ}$ semestre $16(7,5 \%)$, o $5^{\circ}$ semestre $38(17,8 \%), 14(6,5 \%)$ o $6^{\circ}$ semestre, $47(22 \%)$ o $7^{\circ}$ semestre do curso, o $8^{\circ}$ semestre $10(4,7 \%)$, o $9^{\circ}$ semestre $30(14 \%)$ e o $10^{\circ}$ semestre 24 (11,2\%). Foram 116 (54,2\%) participantes do Estado da Bahia, 71 (33,2\%) do Estado de São Paulo, 13 (6,1\%) de Pernambuco, 7 (3,3\%) do Paraná, 6 (2,8\%) de Minas Gerais e $1(0,5 \%)$ do Rio Grande do Sul.

\section{Instrumento}

Foi criado um instrumento visando avaliar a ansiedade em universitários do curso de Psicologia. De acordo com Pasquali (1998), existem três procedimentos básicos para a construção de uma escala psicométrica: o procedimento teórico, o empírico e o analítico. No procedimento teórico, procuraram-se na literatura as definições do construto ansiedade, bem como as derivações ansiedade-traço e ansiedade-estado. Na fase empírica foram elaborados 44 itens divididos em duas partes. Na primeira parte, foi solicitado aos respondentes que marcassem dentro de 20 itens ligados a sintomas relacionados à ansiedade sentidos no último semestre, marcando em uma escala Likert de cinco pontos (1 - Discordo totalmente, 2 - Discordo parcialmente, 3 - Indiferente, 4 - Concordo parcialmente, 5 - Concordo totalmente). $\mathrm{Na}$ segunda parte, foram apresentados 24 itens, divididos em 4 fatores, com base nas leituras dos estudos encontrados sobre o tema. O primeiro deles foi "Questões Universitárias", que reunia itens referentes à dinâmica da vida acadêmica, de uma forma geral, tal como a carga horária de aulas e a quantidade de material para estudar. O segundo fator diz respeito ao "Mercado de Trabalho" e continha itens ligados à insegurança quanto à formação e a atuação profissional. No fator "Expectativa Social" buscou-se saber como o (a) respondente percebia a expectação que a sociedade possuía no que se refere à sua formação profissional. O quarto fator foi a "Expectativa Pessoal" com itens para avaliar como o (a) respondente concebia o curso, a sua escolha profissional e a expectativa familiar para a conclusão do curso. Em todos esses itens foi 
solicitado que o (a) respondente marcasse na escala de cinco pontos (Likert) o grau de concordância com eles.

\section{Procedimento de coleta e análise de dados}

Os dados foram coletados por meio da utilização de plataforma do Google Forms, uma ferramenta digital usada para coletar dados em pequenas ou grandes quantidades, mediante a criação de questionários pelos usuários. Informados acerca dos objetivos da pesquisa, bem como a utilização das respostas no trabalho em questão e a possibilidade da publicação de futuros artigos sobre o tema, por meio de uma versão digital do TCLE (Termo de Consentimento Livre e Esclarecido), foi solicitado que os respondentes marcassem a opção "concordo" para poderem prosseguir na pesquisa. 0 tempo de resposta foi de 15 minutos, e a coleta foi realizada no período de uma semana. Os dados foram analisados por meio do programa Statistical Package for the Social Sciences - SPSS v.22, com análises estatísticas descritivas (média, desvio padrão, pontuações mínima e máxima) e inferenciais (análise fatorial exploratória, teste t e ANOVA).

\section{Resultados}

Primeiramente, optou-se por analisar as propriedades psicométricas da escala para, depois, verificar os níveis de ansiedade, assim como buscar possíveis diferenças entre os gêneros, tipo de instituição, semestre e região de moradia. Assim, abaixo estão os dados de uma Análise de Componentes Principais (ACP) realizada para verificar se os itens se agruparam como pretendido na construção do instrumento.

Após a obtenção dos dados foi realizado um primeiro passo estatístico a fim de verificar se a matriz de correlação dos dados é passível de fatoração com a obtenção do critério Kaiser-Meyer-Olkin (KMO). Tal índice indica a quão adequada é a aplicação da Análise Fatorial Exploratória (AFE) para o conjunto de dados (HAIR et al., 2005). valor encontrado foi de 0,91, considerado como excelente (DAMÁSIO, 2012), demonstrando que o uso da AFE é apropriado para melhor compreensão dos dados da pesquisa.

O segundo elemento de verificação da possibilidade de fatoração dos dados foi o teste de esfericidade de Bartlett, que verifica a verossimilhança entre a matriz dos dados de pesquisa e a matriz identidade, revelando se é possível se estabelecer uma correlação entre os itens pesquisados. Para esta pesquisa a significância foi menor que 
0,001 $(<0,05)$, indicando que a matriz é favorável ao estabelecimento de correlações (HAIR et al., 2005). Na Tabela 1 seguem os resultados da análise.

Tabela 1 - Itens e carga fatorial em cada um dos fatores.



Fonte: Elaborado pela autora.

A análise apontou oito fatores que não concordam com o proposto pela teoria traço-estado utilizada para a construção dos itens, assim optou-se por fixar dois fatores, com o objetivo de que os itens correspondentes ao traço e ao estado se dividissem e foi o que aconteceu, podendo ser visualizado na Tabela 1. Apenas o item 
14 da parte "estado" não carregou no fator, sendo considerados itens com carga superior a 0,30. Assim, decidiu-se pela exclusão do item. A segunda parte da escala, referente à ansiedade estado no contexto universitário foi proposta em quatro fatores, a saber, Contexto Universitário, Mercado de Trabalho, Expectativa Social e Expectativa Pessoal. Para verificar se os itens se agruparam nos fatores propostos foi realizada outra ACP apenas com os itens da segunda parte. O resultado está na Tabela 2.

Com a análise fatorial chegou-se à conclusão de que a melhor solução é a de dois fatores, sendo um deles relacionado às questões do contexto universitário e outro relacionado ao contexto do mercado de trabalho, sendo que no primeiro fator ficaram 7 itens e no segundo 5 . Os dados são passíveis de fatoração visto que o KMO encontrado foi 0,85 e o teste de esfericidade de Bartlett foi significativo estatisticamente $(<0,0001)$.

Tabela 2 - Itens e carga fatorial da parte 2, ansiedade estado.

\begin{tabular}{lcc}
\hline & \multicolumn{3}{c}{ Componente } \\
\cline { 2 - 3 } & $\mathbf{1}$ & $\mathbf{2}$ \\
\hline ansiedade-estado_22 & 0,905 & \\
ansiedade-estado_5 & 0,894 & \\
ansiedade-estado_3 & 0,688 & \\
ansiedade-estado_9 & 0,616 & \\
ansiedade-estado_19 & 0,539 & \\
ansiedade-estado_16 & 0,475 & 0,900 \\
ansiedade-estado_8 & 0,405 & 0,878 \\
ansiedade-estado_20 & & 0,871 \\
ansiedade-estado_21 & & 0,581 \\
ansiedade-estado_23 & & 0,513 \\
ansiedade-estado_15 & & \\
ansiedade-estado_2 & & \\
\hline
\end{tabular}

Fonte: Elaborado pela autora.

Após a adequação da escala dá-se o seguimento para os demais objetivos deste trabalho, a saber: verificar diferenças entre os gêneros; tipo de instituição; semestre e região de moradia. Para a primeira parte da escala, ansiedade traço, não foi encontrada diferença significativa para o gênero $\left(M_{f}=2,91 ; M_{m}=2,62\right)$, e o mesmo aconteceu com a variável semestre, que foram agrupados em início (1으, 2으, 3으 e 4으) e 
fim $(70,8 \circ$, 9ㅇ e 10) , as médias foram, 2,72 e 2,81, respectivamente. Com relação à região de moradia, quando agrupados em Nordeste (Bahia e Pernambuco) e Sudeste/Sul (São Paulo, Minas Gerais, Paraná e Rio Grande do Sul) não foi encontrada diferença, mas ao comparar somente os estudantes da Bahia e de São Paulo houve diferença, mostrando os universitários de São Paulo mais ansiosos $(M=3,00)$ quando comparados aos da Bahia $(M=2,71)$, resultado esse estatisticamente significativo $(p=0,05)$. Para o tipo de instituição, particular e pública, observou-se que os discentes das universidades particulares são mais ansiosos $(M=3,04)$ quando comparados com os da pública $(M=2,59)$, resultado estatisticamente significativo $(p<0,001)$.

Para a segunda parte da escala, ansiedade estado, foram realizadas as mesmas análises. Para o gênero foram encontradas diferenças significativas nos dois fatores, sendo as mulheres com maior pontuação comparadas aos homens ( $F 1, M_{f}=3,52$, $M_{m}=3,04 ; F 2, M_{f}=4,25, M_{m}=3,80$ ). Quanto ao tipo de instituição não foi encontrada diferença estatisticamente significativa. Com relação ao semestre, a diferença encontrada foi para o fator 2 , com médias maiores para os alunos do final do curso $\left(M_{i}=3,75, M_{f}=4,27\right)$. Para a região de moradia não foi encontrada diferença significativa entre os estados dos participantes.

\section{Discussão e considerações finais}

Este trabalho teve como objetivo construir e fazer estudos iniciais de validação de uma escala para avaliação da ansiedade, tendo como público-alvo estudantes do curso de Psicologia. Também foram objetivos deste estudo verificar possíveis diferenças nos níveis de ansiedade para as seguintes variáveis: gênero, tipo de instituição, semestre e região de moradia.

Primeiramente foram estudadas as propriedades psicométricas da escala construída. Entende-se que os resultados aqui obtidos necessitam de mais aprimoramento, como, por exemplo, a realização de análises estatísticas mais robustas, com ampliação da amostra para outros cursos e estados, bem como análise da fidedignidade da escala. Contudo, percebeu-se que ambas as partes da escala, a saber, ansiedade traço e estado, apresentaram índices positivos e significativos com relação à fatoração do instrumento. A escala ficou composta por 32 itens, assim distribuídos, 20 itens na primeira parte, ansiedade traço, e 12 itens na segunda, ansiedade estado. Para a segunda parte foram formados dois fatores, o primeiro 
relacionado a questões do contexto universitário, com 7 itens, e o segundo com questões sobre o mercado de trabalho, com 5 itens.

Após a adequação psicométrica, foram verificadas as possíveis diferenças para as variáveis elencadas. Observou-se que os resultados foram diferentes para cada parte da escala. Dessa maneira, para as variáveis gênero e semestre não foram encontradas diferenças estatisticamente significativas, no que se refere à ansiedade traço. O estudo de Vasconcelos et al. (2015) objetivou determinar a prevalência de sintomas de ansiedade em estudantes de Medicina e avaliar fatores associados. Participaram do estudo 237 estudantes de uma universidade pernambucana, sendo 154 do sexo feminino e 83 do sexo masculino. Dos fatores associados à sintomas de ansiedade não foram encontradas diferenças estatisticamente significativas em relação ao sexo, procedência, morar com a família, ter parceria fixa, ter atividade extracurricular remunerada e uso de drogas ilícitas. Em um outro trabalho, Bruch, Carneiro e Jornada (2009) tiveram como objetivo avaliar a prevalência de sintomas depressivos e ansiosos nos acadêmicos do curso de medicina, de uma Universidade do sul do Brasil. Foram avaliados 233 estudantes do 3 o ao 8 o semestre, sendo que para analisar a sintomatologia depressiva foi aplicado o Inventário de Depressão de Beck e para a sintomatologia ansiosa o Inventário de Ansiedade Estado-Traço. De maneira geral, os autores concluíram que não há correlação entre sintomas depressivos e ansiosos e semestres específicos.

Quando realizada a comparação das pontuações dos sujeitos pelo tipo de instituição de ensino em que estudam, percebeu-se que os alunos de universidades particulares apresentaram maiores médias comparados aos de universidades públicas. Não foram encontrados outros estudos que possibilitassem uma discussão mais ampla desse resultado. Contudo, acredita-se que os alunos de instituições particulares possuem particularidades, como, por exemplo, o pagamento do curso que realizam, que pode fazer com que estejam mais ansiosos.

Ainda para a ansiedade traço, no que tange à região de moradia dos participantes, os estudantes de São Paulo apresentaram índices mais elevados de ansiedade do que os do estado da Bahia. Corroborando com este resultado, o trabalho de Gama et al. (2008), que buscou avaliar ansiedade - traço em universitários de diferentes cursos de uma universidade do estado de Sergipe, encontrou índices significativamente menores de ansiedade nos indivíduos aracajuanos quando comparados com os indivíduos de São Paulo. Isso pode ser influenciado pelo fato deste 
estado possuir uma dinâmica mais agitada, quando comparada às cidades de Salvador e Aracaju, ambas localizadas no nordeste do país, impactando, assim, diretamente na vida dos estudantes universitários. Já no estudo de Marques e Tavano (2004) denominado "Ansiedade Traço-Estado em estudantes universitários" procuraram descrever os escores de ansiedade traço-estado da amostra composta por 37 estudantes universitários dos cursos de Odontologia e Fonoaudiologia de uma Universidade de São Paulo. No momento da aplicação do inventário, no que se refere aos escores da escala Estado, a maioria apresentou nível baixo de ansiedade, já na escala Traço a maioria dos indivíduos apresentou nível de ansiedade moderado mostrando resultados diferentes.

Com relação à segunda parte da escala, como observado nos resultados, ansiedade - estado, as diferenças buscadas para tipo de instituição e região de moradia não foram encontradas. Para a variável gênero, as mulheres apresentaram níveis maiores de ansiedade comparadas aos homens. Isso também foi encontrado no estudo de Carvalho et al. (2015), em que foi analisado o índice de ansiedade em estudantes ingressantes e concluintes de uma instituição de ensino superior, as mulheres também se mostraram mais ansiosas. Resultado que se repete no trabalho de Medeiros e Bittencourt (2017), já que afirmam que as mulheres são mais ansiosas em relação aos homens. Outra pesquisa que mostrou o mesmo foi a de Reis, Miranda e Freitas (2017), pois também encontraram que mulheres são mais ansiosas do que os homens, quanto à ansiedade-estado. Existe uma questão social por trás da variável "gênero", quando se entende que condições profissionais oferecidas às mulheres possuem uma disparidade em relação aos homens. Consequentemente, os desafios apresentados às mulheres são maiores, e a consolidação de uma carreira pode ser mais difícil, levandoas então a apresentar comportamentos mais ansiosos que os homens (CARVALHO et al., 2015).

Quanto à variável semestre foi constatado que os estudantes dos semestres finais se revelaram mais ansiosos do que os estudantes de semestres iniciais para o fator que avalia questões relativas ao mercado de trabalho. Em seu estudo, Carvalho et al. (2015) encontraram que os concluintes eram mais ansiosos em relação aos ingressantes. Acredita-se que isso se justifique pelo fato de esses estudantes estarem mais próximos da conclusão do curso e, consequentemente, mais preocupados com questões profissionais e o mercado de trabalho. Entretanto, no estudo de Costa e Oliveira (2012), que objetivou revelar a ausência ou presença de indicadores de 
estresse e ansiedade em estudantes de psicologia, os resultados indicaram que os iniciantes apresentaram sintomas de ansiedade em níveis mais altos do que a os concluintes; entretanto, os concluintes apresentaram níveis mais elevados de estresse. Pôde-se inferir, então, que estudantes ingressantes possuem grande expectativa com relação ao curso.

Conclui-se que os diferentes tipos de ansiedade, traço e estado, possuem diferentes características que podem se apresentar de diversas formas nos universitários pesquisados. Enquanto para a ansiedade traço as diferenças aconteceram para as variáveis "tipo de instituição" e "região de moradia", para a ansiedade estado foram o "gênero" e o "semestre cursado". Acredita-se que as especificidades de cada tipo de ansiedade respondem essas diferenças, visto que a ansiedade traço está ligada à personalidade do sujeito, ou seja, o indivíduo é ansioso independentemente de cursar ou não a universidade. Assim, observa-se que os estudantes de universidades particulares possuem tais características que podem gerar mais ansiedade, aspectos relacionados aos Estados dos participantes também podem corroborar essa ideia. $\mathrm{Na}$ ansiedade estado, que confere ansiedade momentânea ao sujeito, acredita-se que as mulheres podem vivenciar mais situações de ansiedade em relação aos homens, e o fato dos concluintes se mostrarem mais ansiosos com relação ao mercado de trabalho faz sentido visto que logo estarão à procura de emprego, com a finalização do curso.

Mesmo o estudo tendo atingido os objetivos propostos, algumas limitações foram enfrentadas. Uma delas é a aplicação da escala ter sido realizada por meio da internet, o que não possibilita verificar se os participantes entenderam os objetivos do trabalho, assim como os itens apresentados. O tempo curto para a coleta dos dados também se mostra como limitação, visto que a amostra seria mais ampla se o tempo fosse maior e, talvez, outros estados pudessem ser contemplados, assim como mais estudantes dos estados participantes.

Sugere-se que outros estudos sejam realizados com a escala, envolvendo mais participantes e também de outros cursos que não só a Psicologia, a fim de verificar como estão os níveis de ansiedade desses alunos. Também pode a ansiedade estar relacionada a outros aspectos do curso, como por exemplo a adaptação acadêmica ou a motivação para aprender, temas estes que podem mostrar alguns problemas enfrentados pelos estudantes, gerando, assim, ansiedade. 


\section{Referências}

AMERICAN PSYCHIATRIC ASSOCIATION. APA. Manual diagnóstico e estatístico de transtornos mentais: DSM-V. 5. ed. Porto Alegre: Artmed, 2014.

ARIÑO, Daniela Ornellas; BARDAGI, Marúcia Patta. Relação entre fatores acadêmicos e a saúde mental de estudantes universitários. Revista psicologia em pesquisa, v. 12, n. 3, p. 44-52, 2018. Disponível em:

http://pepsic.bvsalud.org/scielo.php?script=sci_abstract\&pid=S1982-

12472018000300005\&Ing=en\&nrm=iso\&tlng=pt. Acesso em: 17 ago. 2020.

BEZERRA, Marilia Lopes Oliveira; SIQUARA, Gustavo Marcelino; ABREU, José Neander Silva. Relação entre os pensamentos ruminativos e índices de ansiedade e depressão em estudantes de psicologia. Revista Psicologia, Diversidade e Saúde, v. 7, n. 2, p. 235-244, 2018. Disponível em:

https://www5.bahiana.edu.br/index.php/psicologia/article/view/1906/1828. Acesso em: 16 ago. 2020.

BRANDTNER, Maríndia; BARDAGI, Marucia. Sintomatologia de depressão e ansiedade em estudantes de uma universidade privada do Rio Grande do Sul. Gerais: Revista Interinstitucional de Psicologia, v. 2, n. 2, p. 81-91, 2009. Disponível em: http://pepsic.bvsalud.org/scielo.php?script=sci_arttext\&pid=S198382202009000200004 . Acesso em: 16 ago. 2020.

BRUCH, Tatiana Pizzolotto; CARNEIRO, Ellis Alves; JORNADA, Luciano Kurtz. Presença de sintomas psiquiátricos em estudantes de medicina de Universidade do sul do Brasil. Arquivos Catarinenses de Medicina, v. 38, n. 4, p. 61-5, 2009.

CARDOZO, Mayara Quadros et al. Fatores associados à ocorrência de ansiedade dos acadêmicos de Biomedicina. Saúde e Pesquisa, v. 9, n. 2, p. 251-262, 2016.

CARVALHO, Eliane Alicrim de et al. Índice de ansiedade em universitários ingressantes e concluintes de uma instituição de ensino superior. Ciência, Cuidado e Saúde, v 14, n. 3, p. 1290-1298, 2015. Disponível em:

https://pesquisa.bvsalud.org/portal/resource/pt/biblio-1121148. Acesso em: 16 ago. 2020.

CHAVES, Erika de Cássia Lopes et al. Ansiedade e espiritualidade em estudantes universitários: um estudo transversal. Revista brasileira de enfermagem, v. 68, n. 3, p. 504-509, 2015. Disponível em:

https://www.scielo.br/j/reben/a/MBK4J58g4f9Jw3nrXGRLHGx/?lang=pt. Acesso em: 16 ago. 2020.

COSTA, Kercia Mirely Vieira et al. Ansiedade em universitários na área da saúde. In: CONGRESSO BRASILEIRO DAS CIÊNCIAS DA SAÚDE (COMBRACIS), 2., 2017, 
Campina Grande. Anais [...]. Campina Grande: COMBRACIS, 2017. Disponível em: https://www.editorarealize.com.br/editora/anais/conbracis/2017/TRABALHO_EV071 _MD1_SA13_ID592_14052017235618.pdf. Acesso em: 16 ago. 2020.

COSTA, Victor Cesar Amorim; OLIVEIRA, Andréa Olimpio. Estudo comparativo dos indicadores de sintomas de estresse e ansiedade entre estudantes entrantes e concluintes do curso de Psicologia. In: SIMPÓSIO DE PRODUÇÃO ACADÊMICA (SIMPAC), 4., 2012, São Paulo. Anais [...]. São Paulo: SIMPAC, 2012. Disponível em: https://academico.univicosa.com.br/revista/index.php/RevistaSimpac/article/view/2 13. Acesso em: 16 ago. 2020.

DAMÁSIO, Bruno Figueiredo. Uso da análise fatorial exploratória em psicologia. Avaliaçao Psicologica, v. 11, n. 2, p. 213-228, 2012.

FERNANDES, Márcia Astrês et al. Prevalência de sintomas ansiosos e depressivos em universitários de uma instituição pública. Revista Brasileira de Enfermagem, v. 71, 2018.

GAMA, Marcel Magalhães Alves et al. Ansiedade-traço em estudantes universitários de Aracaju (SE). Revista de Psiquiatria do Rio Grande do Sul, v. 30, n. 1, p. 19-24, 2008. Disponível em: http://www.scielo.br/scielo.php?script=sci_arttext\&pid=S010181082008000100007\&lng=en\&nrm=iso. Acesso em: 17 nov. 2019.

HAIR, Joseph F. et al. Análise multivariada de dados. Porto Alegre: Bookman, 2005. HOLLANDER, Eric; SIMEON, Dalphane. Transtornos de ansiedade. Porto Alegre: Artmed, 2004.

MARCHI, Katia Colombo et al. Ansiedade e consumo de ansiolíticos entre estudantes de enfermagem de uma universidade pública. Revista Eletrônica de Enfermagem, v. 15, n. 3, p. 729-37, 2013.

MARGIS, Regina et al. Relação entre estressores, estresse e ansiedade. Revista de Psiquiatria do Rio Grande do Sul, v. 25, p. 65-74, 2003. Disponível em: https://www.scielo.br/j/rprs/a/Jfqm4RbzpJhbxskLSCzmgjb/?lang=pt. Acesso em: 17 nov. 2019

MARQUES, Luciana Corrêa; TAVANO, Liliam D.'Aquino. Ansiedade traço-estado em estudantes universitários. Revista Científica Eletrônica de Psicologia, v. 2, p. 1-3, 2004. Disponível em:

http://faef.revista.inf.br/imagens_arquivos/arquivos_destaque/EvSU73xXdkTTZsP_2 013-4-30-12-10-9.pdf. Acesso em: 17 nov. 2019

MEDEIROS, Palloma Prates; BITTENCOURT, Felipe Oliveira. Fatores associados à ansiedade em estudantes de uma faculdade particular. Id on Line Revista de Psicologia, v. 10, n. 33, p. 42-55, 2017. 
PINTO, Cândida; PAIS-RIBEIRO, José Luís. Construção de uma escala de avaliação da espiritualidade em contextos de saúde. Arquivos de medicina, v. 21, n. 2, p. 47-53, 2007.

PASQUALI, Luiz. Princípios de elaboração de escalas psicológicas. Revista de psiquiatria clínica, v. 25, n. 5, p. 206-213, 1998.

REIS, Clara Figueira; MIRANDA, Gilberto José; FREITAS, Sheizi Calheira. Ansiedade e desempenho acadêmico: um estudo com alunos de ciências contábeis. Advances in Scientific and Applied Accounting, v. 10, n. 3, p. 319-333, 2017.

SADOCK, Benjamin J.; SADOCK, Virginia A.; RUIZ, Pedro. Compêndio de psiquiatria: ciência do comportamento e psiquiatria clínica. Porto Alegre: Artmed Editora, 2016. SKINNER, Burrhus Frederic. Ciência do comportamento humano. São Paulo: Martins Fontes, 1989.

VASCONCELOS, Tatheane Couto de et al. Prevalência de sintomas de ansiedade e depressão em estudantes de medicina. Revista Brasileira de Educação Médica, v. 39, n. 1, p. 135-142, 2015.

Recebido em: set. 2020

Aprovado em: maio 2021 